organic communications

\title{
Succinic acid: a novel and efficient organo-catalyst for synthesis of a-amino nitriles under solvent free condition
}

\author{
Ramesh S. Ghogare ${ }^{1 *}$ \\ Department of Chemistry, B. N. N. College Bhiwandi, Dist-Thane, Maharashtra, India-421305
}

(Received August 24, 2020; Revised September 06, 2020; Accepted September 11, 2020)

\begin{abstract}
A simple, efficient and eco-friendly general protocol has been developed for the synthesis of threecomponent reaction methodology of Strecker's $\alpha$-amino nitriles by using various aldehyde or ketone, amines and trimethylsilyl cyanide (TMS-CN). In this methodology, succinic acid is used as a novel and efficient organocatalyst in catalytic amount. This method was carried out under solvent free condition at room temperature for preparation of variety of $\alpha$-amino nitriles derivatives in excellent yields. The present method provides significant advantages of organo-catalyst such as inexpensive, highly stable, environmentally benign and commercially available as well as solvent free reaction conditions and high conversions of the products with excellent yield.
\end{abstract}

Keywords: Strecker reaction; $\alpha$-amino nitriles ; TMS-CN; succinic acid; organo-catalyst; solvent-free. @2020 ACG Publication. All right reserved.

\section{Introduction}

In the recent years, an organocatalysis has been gained more attraction worldwide in the research of advanced organic chemistry because of their important characteristics including metal-free atmosphere, operational ease, reduced-cost and relatively less harmfulness ${ }^{1-6}$. It has been different mode of catalysis such as Brønsted acid-base catalysis, Lewis acid-base catalysis, Nucleophilic catalysis and Redox catalysis. An organo-catalyst is a simple organic molecule which is help to chemical transformation by activation of chemical reagents through various manners with increasing the mildness of the reaction conditions. One of these Brønsted acid catalysts, succinic acid has been used in the building block ${ }^{7}$ for production of industrial chemicals ${ }^{8}$, plasticizers ${ }^{9}$, polyesters ${ }^{10}$, solvents ${ }^{11}$ and biopolymers ${ }^{12}$. In the past years, for the efficient and practical chemical reaction condition, some methodologies have been reported by using succinic acid as catalysts in synthesis of $\alpha$-amino phosphonates ${ }^{13-14}$ and Dihydropyridines ${ }^{15}$. Recently, we also reported succinic acid as catalyst for the synthesis of 3,4-disubstituted isoxazol-5(4H)-ones ${ }^{16}$. Herein, we described applicability of succinic acid as a readily available, efficient acidic organo-catalyst for the synthesis of $\alpha$-amino nitriles.

The Strecker reaction ${ }^{17-19}$ is the first multi-component reaction (MCR) reported for the synthesis of $\alpha$-amino nitriles through the condensation of three-component of amine, cyanide and aldehyde in 1850. $\alpha$-amino nitriles are the key important bi-functional intermediate in the synthesis of versatile precursors of $\alpha$-amine acids ${ }^{20-21}$, biologically active molecules ${ }^{22-26}$ and chiral building blocks ${ }^{27-30}$. In the view of the emerging importance of $\alpha$-amino nitriles, many of the researchers led to the development of efficient protocols using various catalysts, reagents and conditions. Recently, numerous methods have been developed for the synthesis of Strecker's $\alpha$-aminonitriles by using various catalysts such as Organo-catalysts ${ }^{31-38}$, Heterogeneous Brønsted acids ${ }^{39-45}$, Heterogeneous Lewis acids ${ }^{46-52}$, Homogeneous

\footnotetext{
*E-Mail: rsghogare05@gmail.com
} 
Lewis acids ${ }^{53-59}$, Metals or Metal based complexes ${ }^{60-62}$, Ionic liquids ${ }^{63-67}$ as well as Polymeric Materials $^{68-69}$. In similar manner, different Solvent systems ${ }^{70-73}$ and Cyanide sources ${ }^{74-78}$ are also reported in the literature. Many of the reported methods involve one or two limitations such as, use of expensive reagents, harsh reaction conditions, extended reaction times, tedious workup procedures and low yields. In some cases, the catalysts are decomposed or deactivated by amines. Therefore, there is scope to develop efficient, milder conditions and environmental benign green chemical processes are major challenges for researcher in organic synthesis. As part of our ongoing research, to develop a novel methodology using alternative protocols ${ }^{79-80}$ and by considering the importance $\alpha$-amino nitriles, herein, we report, a simple and efficient synthesis of $\alpha$-amino nitriles by using succinic acid as an organocatalyst under solvent free condition. Succinic acid is mild, inexpensive, highly stable, environmentally benign and commercially available compound. In order to minimize toxicity of cyanide reagent, in this method TMS-CN is used as cyanide source for nucleophilic addition to the imines.

\section{Experimental}

\subsection{Chemical Material and Apparatus}

Reagents and solvents were purchased from commercial sources and used without further purification. ${ }^{1} \mathrm{H}$ NMR spectra were recorded on Gemini-300 spectrometer in $\mathrm{CDCl}_{3}$ using TMS as an internal standard and IR spectra were recorded on a Bruker FT-IR spectrophotometer using neat or $\mathrm{KBr}$ disk. Mass spectra were recorded on a Finnigan MAT 1020 mass spectrometer operating at $70 \mathrm{eV}$. Melting points were determined with Buchi R-535 apparatus are uncorrected.

\subsection{General Procedure}

A mixture of aromatic aldehyde or ketone $(1 \mathrm{mmol})$, amines $(1 \mathrm{mmol})$ and TMS-CN $(1.2 \mathrm{mmol})$ was stirred in presence of succinic acid $(15 \% \mathrm{~mol})$ as catalyst under solvent free condition at room temperature. After completion of reaction monitored by thin layer chromatography, $10 \mathrm{ml}$ water was added to the mixture and the product was extracted with ethyl acetate $(2 \times 10 \mathrm{ml})$. The combined ethyl acetate extract was washed with brine, dried over $\mathrm{Na}_{2} \mathrm{SO}_{4}$ and the crude product was purified concentrated under reduced pressure by column chromatography using silica gel (60-120 mesh) by eluting with ethyl acetate-hexane mixture (2:8). All the pure products were confirmed by their spectroscopy data.

\subsection{Spectral Data of Synthesized Compounds}

2-phenyl-2-(phenylamino)acetonitrile (4a): White solid; m.p.78-80 ${ }^{\circ} \mathrm{C}\left({ }^{\mathrm{Lit}[38]} 81-83{ }^{\circ} \mathrm{C}\right)$; IR $(\mathrm{KBr}) \bar{v}=$ $3360,3032,2968,2257,1585,1476,1291,1176, \mathrm{~cm}^{-1} .{ }^{1} \mathrm{H}$ NMR $\left(300 \mathrm{MHz}, \mathrm{CDCl}_{3}\right): \delta=3.98(\mathrm{~d}, 1 \mathrm{H}, J$ $=8.4 \mathrm{~Hz}, \mathrm{NH}), 5.50(\mathrm{~d}, 1 \mathrm{H}, J=8.4 \mathrm{~Hz}, \mathrm{CH}-\mathrm{CN}), 6.81(\mathrm{~d}, 2 \mathrm{H}, J=8.2 \mathrm{~Hz}, \mathrm{ArH}), 6.93(\mathrm{t}, 1 \mathrm{H}, J=7.5 \mathrm{~Hz}$, $\mathrm{ArH}), 7.26(\mathrm{t}, 2 \mathrm{H}, J=7.5 \mathrm{~Hz}, \mathrm{ArH}), 7.44-7.56(\mathrm{~m}, 3 \mathrm{H}, \mathrm{ArH}), 7.78-7.86(\mathrm{~m}, 2 \mathrm{H}, \mathrm{ArH}) \mathrm{ppm} .{ }^{13} \mathrm{C} \mathrm{NMR}$ $\left(75 \mathrm{MHz}, \mathrm{CDCl}_{3}\right): \delta: 50.31,114.6,118.5,120.5,127.3,128.7,129.2,133.7,144.2 \mathrm{ppm}$. ESIMS: $\mathrm{m} / \mathrm{z}$ : $209\left(\mathrm{M}^{+1}\right)$.

2-((4-Nitrophenyl)amino)-2-phenylacetonitrile (4b): White solid; m.p. $125-127{ }^{\circ} \mathrm{C}\left({ }^{\mathrm{Lit}[70]} 127-129{ }^{\circ} \mathrm{C}\right)$; $\operatorname{IR}(\mathrm{KBr}) \bar{v}=3330,2995,2246,1535,1423,1364,1270,1156 \mathrm{~cm}^{-1} .{ }^{1} \mathrm{H}$ NMR $\left(300 \mathrm{MHz}, \mathrm{CDCl}_{3}\right): \delta=$ $4.76(\mathrm{~d}, 1 \mathrm{H}, J=7.1 \mathrm{~Hz},-\mathrm{NH}), 5.51(\mathrm{~d}, 1 \mathrm{H}, J=7.3 \mathrm{~Hz}, \mathrm{CH}-\mathrm{CN}), 6.77(\mathrm{~d}, 2 \mathrm{H}, J=9.2 \mathrm{~Hz}, \mathrm{ArH}), 7.44-$ $7.56(\mathrm{~m}, 5 \mathrm{H}, \mathrm{ArH}), 8.12(\mathrm{~d}, 2 \mathrm{H}, J=7.2 \mathrm{~Hz}, \mathrm{ArH}) \mathrm{ppm} .{ }^{13} \mathrm{C}$ NMR $\left(75 \mathrm{MHz}, \mathrm{CDCl}_{3}\right): \delta=48.4,116.3$, 116.6, 127.8, 128.9, 129.5, 133.3, 136.3, $151.6 \mathrm{ppm}$. ESIMS: $m / z: 254\left(\mathrm{M}^{+1}\right)$.

2-((4-fluorophenyl)amino)-2-phenylacetonitrile (4c): Yellow solid; m.p. $88-90{ }^{\circ} \mathrm{C}\left({ }^{\mathrm{Lit}}[70]\right.$ 88-90 $\left.{ }^{\circ} \mathrm{C}\right)$; IR $(\mathrm{KBr}) \bar{v}=3348,3064,2242,1579,1466,1293,1196 \mathrm{~cm}^{-1} .{ }^{1} \mathrm{H}$ NMR $\left(300 \mathrm{MHz}, \mathrm{CDCl}_{3}\right): \delta=3.96$ (brs, $1 \mathrm{H}, J=5.6 \mathrm{~Hz},-\mathrm{NH}), 5.36(\mathrm{~d}, 1 \mathrm{H}, J=5.6 \mathrm{~Hz},-\mathrm{CH}-\mathrm{CN}), 6.69-6.77(\mathrm{~m}, 2 \mathrm{H}, \mathrm{ArH}), 6.96(\mathrm{t}, 2 \mathrm{H}, J=8.4$ $\mathrm{Hz}, \mathrm{ArH}), 7.42-7.49(\mathrm{~m}, 3 \mathrm{H}, \mathrm{ArH}), 7.54-7.62(\mathrm{~m}, 2 \mathrm{H}, \mathrm{ArH}) \mathrm{ppm} ;{ }^{13} \mathrm{C} \mathrm{NMR}\left(75 \mathrm{MHz}, \mathrm{CDCl}_{3}\right): \delta: 50.0$, 116.7, 120.3, 120.6, 127.0, 127.5, 133.2, 143.4, 151.3 ppm. ESIMS: $m / z: 227\left(\mathrm{M}^{+1}\right)$. 
2-((4-methoxyphenyl)amino)-2-phenylacetonitrile (4d): White solid; m.p. $74-76{ }^{\circ} \mathrm{C}\left({ }^{\mathrm{Lit}}[70] 75-77{ }^{\circ} \mathrm{C}\right)$; IR $(\mathrm{KBr}) \bar{v}=3362,2946,2236,1585,1488,1435,1274,1238, \mathrm{~cm}^{-1} .1 \mathrm{H}$ NMR $\left(300 \mathrm{MHz}, \mathrm{CDCl}_{3}\right): \delta=3.75$ $\left(\mathrm{s}, 3 \mathrm{H},-\mathrm{OCH}_{3}\right), 3.98(\mathrm{~d}, 1 \mathrm{H}, J=5.6 \mathrm{~Hz},-\mathrm{NH}), 5.34(\mathrm{~s}, 1 \mathrm{H},-\mathrm{CH}-\mathrm{CN}), 6.76(\mathrm{~d}, 2 \mathrm{H}, J=9.0 \mathrm{~Hz}, \mathrm{ArH})$, $6.85(\mathrm{~d}, 2 \mathrm{H}, J=9.0 \mathrm{~Hz}, \mathrm{ArH}), 7.42-7.48(\mathrm{~m}, 3 \mathrm{H}, \mathrm{ArH}), 7.60\left(\mathrm{dd}, 2 \mathrm{H}, J_{l}=5.9 \mathrm{~Hz}, J_{2}=1.6 \mathrm{~Hz}, \mathrm{ArH}\right)$ ppm. ${ }^{13} \mathrm{C}$ NMR $\left(75 \mathrm{MHz}, \mathrm{CDCl}_{3}\right): \delta: 48.4,51.7,114.9,116.2,127.4,128.3,134.0,138.5,150.1 \mathrm{ppm}$. ESIMS: $m / z: 238\left(\mathrm{M}^{+}\right)$.

2-morpholino-2-phenylacetonitrile (4e): White solid; m.p. $68-70{ }^{\circ} \mathrm{C}\left({ }^{\mathrm{Lit}[70]} 66-68{ }^{\circ} \mathrm{C}\right), \mathrm{IR}(\mathrm{KBr}) \overline{\mathrm{v}}=3015$, 2949, 2848, 2239, 1601, 1528, 1435, $\left.1274 \mathrm{~cm}^{-1} .1 \mathrm{H} \mathrm{NMR} \mathrm{(300} \mathrm{MHz,} \mathrm{CDCl}_{3}\right): \delta=2.54-2.62$ (m, 4H, 2$\mathrm{CH}_{2}$ ), 3.68-3.78 (m, 4H, 2- $\left.\mathrm{CH}_{2}\right), 4.82(\mathrm{~s}, 1 \mathrm{H},-\mathrm{CH}-\mathrm{CN}), 7.36-7.44(\mathrm{~m}, 3 \mathrm{H}, \mathrm{ArH}), 7.54$ (d, $2 \mathrm{H}, J=7.3$ $\mathrm{Hz}, \mathrm{ArH}) \mathrm{ppm} .{ }^{13} \mathrm{C} \mathrm{NMR}\left(75 \mathrm{MHz}, \mathrm{CDCl}_{3}\right): \delta: 48.8,62.5,66.0,116.0,127.2,127.9,128.5,130.6 \mathrm{ppm}$. ESIMS: $m / z: 203\left(\mathrm{M}^{+1}\right)$.

(4-Methoxyphenyl)-2-(phenylamino)acetonitrile (4f): White solid; m.p. 94-96 ${ }^{\circ} \mathrm{C}\left({ }^{\mathrm{Lit}[53]}\right.$ 95-96 $\left.{ }^{\circ} \mathrm{C}\right)$, IR $(\mathrm{KBr}): \bar{v}=3375,3023,2949,2235,1580,1510,1462,1300, \mathrm{~cm}^{-1} ;{ }^{1} \mathrm{H}$ NMR $\left(300 \mathrm{MHz}, \mathrm{CDCl}_{3}\right): \delta=3.76$ $\left(\mathrm{s}, 3 \mathrm{H},-\mathrm{OCH}_{3}\right), 3.96(\mathrm{~s}, 1 \mathrm{H},-\mathrm{NH}), 5.35(\mathrm{~s}, 1 \mathrm{H},-\mathrm{CH}-\mathrm{CN}), 6.72(\mathrm{~d}, 2 \mathrm{H}, J=8.0 \mathrm{~Hz}, \mathrm{ArH}), 6.88-6.98(\mathrm{~m}$, $3 \mathrm{H}, \operatorname{ArH}), 7.18(\mathrm{~d}, 2 \mathrm{H}, J=8.0 \mathrm{~Hz}, \operatorname{ArH}), 7.60(\mathrm{~d}, 2 \mathrm{H}, J=8.0 \mathrm{~Hz}, \operatorname{ArH}), \mathrm{ppm} ;{ }^{13} \mathrm{C}$ NMR $(75 \mathrm{MHz}$, $\left.\mathrm{CDCl}_{3}\right): \delta=49.1,52.0,113.6,115.3,116.6,120.6,127.8,128.6,129.1,144.8,159.1 \mathrm{ppm}$. ESIMS: $\mathrm{m} / z$ : $239\left(\mathrm{M}^{+1}\right)$.

4-(cyano(phenylamino)methyl)benzonitrile (4g): Pale yellow solid; m.p. $120-122{ }^{\circ} \mathrm{C}\left({ }^{\mathrm{Lit}[53]} 116-118{ }^{\circ} \mathrm{C}\right)$, IR (KBr): 3370, 2868, 2245, 1610, 1530, 1450, $1265 \mathrm{~cm}^{-1} .{ }^{1} \mathrm{H}$ NMR $\left(300 \mathrm{MHz}, \mathrm{CDCl}_{3}\right): \delta=4.70(\mathrm{~d}$, $1 \mathrm{H}, J=7.5 \mathrm{~Hz},-\mathrm{NH}), 5.50(\mathrm{~d}, 1 \mathrm{H}, J=7.6 \mathrm{~Hz}, \mathrm{CH}-\mathrm{CN}), 6.80(\mathrm{~d}, 2 \mathrm{H}, J=7.8 \mathrm{~Hz}, \mathrm{ArH}), 6.96(\mathrm{t}, 1 \mathrm{H}, J$ $=7.4 \mathrm{~Hz}, \mathrm{ArH}), 7.38(\mathrm{t}, 2 \mathrm{H}, J=7.6 \mathrm{~Hz}, \mathrm{ArH}), 7.72(\mathrm{~d}, 2 \mathrm{H}, J=7.4 \mathrm{~Hz}, \operatorname{ArH}), 7.76(\mathrm{~d}, 2 \mathrm{H}, J=7.4 \mathrm{~Hz}$, ArH) ppm. ${ }^{13} \mathrm{C}$ NMR $\left(75 \mathrm{MHz}, \mathrm{CDCl}_{3}\right): \delta=48.6,111.2,111.6,114.6,118.5,120.5,127.5,128.7$, 129.3, 134.9, 143.9 ppm; ESIMS: $m / z: 234\left(\mathrm{M}^{+1}\right)$

3-phenyl-2-(phenylamino)propanenitrile (4h): Pale yellow solid; m.p. $108-110{ }^{\circ} \mathrm{C}, \mathrm{IR}(\mathrm{KBr}): \bar{v}=3324$, 3018, 2924, 2842, 2728, 2228, 1676, 1567, 1462, 1340, $1274 \mathrm{~cm}^{-1} .{ }^{1} \mathrm{H}$ NMR $\left(\mathrm{CDCl}_{3}, 300 \mathrm{MHz}\right): \delta=$ 3.10-3.30 (m, 2H, Ar- $\left.\mathrm{CH}_{2}\right), 4.36(\mathrm{t}, 1 \mathrm{H}, J=6.3 \mathrm{~Hz},-\mathrm{CH}-\mathrm{CN}), 6.76-6.84(\mathrm{~m}, 3 \mathrm{H}, \mathrm{ArH}), 7.30-7.42(\mathrm{~m}$, $5 \mathrm{H}, \mathrm{ArH}), 7.48-7.56(\mathrm{~m}, 2 \mathrm{H}, \mathrm{ArH}) \mathrm{ppm} .{ }^{13} \mathrm{C} \mathrm{NMR}\left(\mathrm{CDCl}_{3}, 75 \mathrm{MHz}\right): \delta=36.8,50.3,114.0,116.3$, 120.4, 126.9, 127.4, 128.6, 129.5, 132.7, 144.6 ppm. ESIMS: $m / z: 245\left(\mathrm{M}^{+23}\right)$.

2-(4-hydroxyphenylamino)-3-phenylpropanenitrile (4i): Pale yellow solid; m.p.. 133-135 ${ }^{\circ} \mathrm{C}\left({ }^{\mathrm{Lit}}[70]\right.$ 136$\left.138{ }^{\circ} \mathrm{C}\right)$, IR (KBr): $\bar{v}=3292,3062,2924,2853,2797,2238,1517,1450,1378,1240 \mathrm{~cm}^{-1} .{ }^{1} \mathrm{H}$ NMR $\left(\mathrm{CDCl}_{3}, 300 \mathrm{MHz}\right): \delta=3.12-3.30\left(\mathrm{~m}, 2 \mathrm{H}, \mathrm{Ar}-\mathrm{CH}_{2}\right), 4.38(\mathrm{t}, 1 \mathrm{H}, J=6.3 \mathrm{~Hz},-\mathrm{CH}-\mathrm{CN}), 4.74$ (brs, $1 \mathrm{H},-$ $\mathrm{OH}), 6.63(\mathrm{~d}, 2 \mathrm{H}, J=8.6 \mathrm{~Hz}, \mathrm{ArH}), 6.75(\mathrm{~d}, 2 \mathrm{H}, J=8.6 \mathrm{~Hz}, \mathrm{ArH}), 7.31-7.44(\mathrm{~m}, 5 \mathrm{H}, \mathrm{ArH}) \mathrm{ppm} .{ }^{13} \mathrm{C}$ NMR $\left(\mathrm{CDCl}_{3}, 75 \mathrm{MHz}\right): \delta=39.0,48.4,116.3,116.6,127.8,128.9,129.5,134.2,138.3,149.8 \mathrm{ppm}$. ESIMS: $m / z: 239\left(\mathrm{M}^{+1}\right)$.

2-(furan-2-yl)-2-(phenylamino)acetonitrile (4j): Brown solid; m.p. $70-72{ }^{\circ} \mathrm{C}\left({ }^{\mathrm{Lit}[44]} 67-69{ }^{\circ} \mathrm{C}\right)$; $\mathrm{IR}(\mathrm{KBr})$ : $\bar{v}=3365,3040,2945,2254,1590,1542,1475,1325 \mathrm{~cm}^{-1} .{ }^{1} \mathrm{H}$ NMR $\left(300 \mathrm{MHz}, \mathrm{CDCl}_{3}\right): \delta=4.04(\mathrm{~d}, 1 \mathrm{H}$, $J=5.6 \mathrm{~Hz}), 5.48(\mathrm{~s}, 1 \mathrm{H},-\mathrm{CH}-\mathrm{CN}), 6.42(\mathrm{~d}, 1 \mathrm{H}, J=3.8 \mathrm{~Hz}, \mathrm{ArH}), 6.58(\mathrm{t}, 1 \mathrm{H}, J=4.7 \mathrm{~Hz}, \mathrm{ArH}), 6.74(\mathrm{~d}$, $2 \mathrm{H}, J=7.8 \mathrm{~Hz}, \mathrm{ArH}), 6.90(\mathrm{t}, 1 \mathrm{H}, J=7.8 \mathrm{~Hz}, \mathrm{ArH}), 7.36(\mathrm{t}, 2 \mathrm{H}, J=7.8 \mathrm{~Hz}, \mathrm{ArH}), 7.52(\mathrm{~d}, 1 \mathrm{H}, J=4.7$ $\mathrm{Hz}, \mathrm{ArH}) \mathrm{ppm} .{ }^{13} \mathrm{C} \mathrm{NMR}\left(75 \mathrm{MHz}, \mathrm{CDCl}_{3}\right): \delta=47.8,111.1,112.0,115.9,121.5,129.5,134.2,136.9$, 145.8, ppm. ESIMS: $m / z: 199\left(\mathrm{M}^{+1}\right)$.

2-(9H-fluoren-2-yl)-2-(phenylamino)acetonitrile (4k): Gray solid; m.p.. $152-154{ }^{\circ} \mathrm{C}\left({ }^{\mathrm{Lit}}{ }^{20]} 152-154{ }^{\circ} \mathrm{C}\right)$; IR (KBr): $\bar{v}=3384,2924,2854,2228,1510,1459,1221 \mathrm{~cm}^{-1} .{ }^{1} \mathrm{H} \mathrm{NMR}\left(\mathrm{CDCl}_{3}, 300 \mathrm{MHz}\right): \delta=3.94(\mathrm{~s}$, $\left.2 \mathrm{H},-\mathrm{CH}_{2}\right), 5.49(\mathrm{~d}, 1 \mathrm{H}, J=8.2 \mathrm{~Hz},-\mathrm{CH}-\mathrm{CN}), 6.81(\mathrm{~d}, 2 \mathrm{H}, J=7.6 \mathrm{~Hz}, \mathrm{ArH}), 6.85-6.95(\mathrm{~m}, 1 \mathrm{H}, \mathrm{ArH})$, 7.24-7.38 (m, 4H, ArH), 7.59 (t, $2 \mathrm{H}, J=8.5 \mathrm{~Hz}, \mathrm{ArH}), 7.78-7.87(\mathrm{~m}, 3 \mathrm{H}, \mathrm{ArH}) \mathrm{ppm} .{ }^{13} \mathrm{C} \mathrm{NMR}\left(\mathrm{CDCl}_{3}\right.$, 
$75 \mathrm{MHz}): \delta=36.8,50.3,114.0,118.4,120.2,120.3,120.4,123.9,125.1,126.0,126.9,127.4,129.5$, 132.0, 140.5, 143.0, 143.4, 144.2, 144.6 ppm. ESIMS: $m / z: 295\left(\mathrm{M}^{-1}\right)$.

2-(9H-fluoren-2-yl)-2-(4-methoxyphenylamino)acetonitrile (4l): Brown solid; m.p.. 140-142 ${ }^{\circ} \mathrm{C}$ (Lit70] 144-146 $\left.{ }^{\circ} \mathrm{C}\right)$; IR (KBr): $\bar{v}=3332,2925,2853,2227,1604,1511,1459,1287,1244 \mathrm{~cm}^{-1} .{ }^{1} \mathrm{H}$ NMR $\left(\mathrm{CDCl}_{3}, 300 \mathrm{MHz}\right): \delta=3.78\left(\mathrm{~s}, 3 \mathrm{H},-\mathrm{OCH}_{3}\right), 3.94\left(\mathrm{~s}, 2 \mathrm{H},-\mathrm{CH}_{2}\right), 5.41(\mathrm{~d}, 1 \mathrm{H}, J=6.4 \mathrm{~Hz},-\mathrm{CH}-\mathrm{CN}), 6.79$ $(\mathrm{d}, 2 \mathrm{H}, J=9.0 \mathrm{~Hz}, \mathrm{ArH}), 6.86(\mathrm{~d}, 1 \mathrm{H}, J=8.2 \mathrm{~Hz}, \mathrm{ArH}), 7.31-7.43(\mathrm{~m}, 3 \mathrm{H}, \mathrm{ArH}), 7.58(\mathrm{t}, 2 \mathrm{H}, J=8.7 \mathrm{~Hz}$, $\mathrm{ArH}), 7.82-7.87(\mathrm{~m}, 2 \mathrm{H}, \mathrm{ArH}) \mathrm{ppm} .{ }^{13} \mathrm{C} \mathrm{NMR}\left(\mathrm{CDCl}_{3}, 75 \mathrm{MHz}\right): \delta=36.8,51.7,55.6,114.9,116.2$, $120.2,120.4,122.1,123.9,124.5,125.1,126.0,126.9,127.4,128.3,132.3,138.5,140.6,143.4,144.2$, 158.6 ppm. ESIMS: $m / z: 327\left(\mathrm{M}^{+1}\right)$.

2-(9H-fluoren-2-yl)-2-morpholinoacetonitrile (4m): White solid; m.p. $175-177{ }^{\circ} \mathrm{C}\left({ }^{\text {Litt70] } 176-178}{ }^{\circ} \mathrm{C}\right)$; IR $(\mathrm{KBr}): \bar{v}=2929,2862,2819,2230,1686,1458,1294 \mathrm{~cm}^{-1} .{ }^{1} \mathrm{H}$ NMR $\left(\mathrm{CDCl}_{3}, 300 \mathrm{MHz}\right): \delta=2.56-2.70$ $\left(\mathrm{m}, 4 \mathrm{H}, 2-\mathrm{CH}_{2}\right), 3.68-3.82\left(\mathrm{~m}, 4 \mathrm{H}, 2-\mathrm{CH}_{2}\right), 3.93\left(\mathrm{~s}, 2 \mathrm{H},-\mathrm{CH}_{2}\right), 4.90(\mathrm{~s}, 1 \mathrm{H},-\mathrm{CH}-\mathrm{CN}), 7.30-7.47(\mathrm{~m}, 2 \mathrm{H}$, $\mathrm{ArH})$, 7.50-7.64 (m, 2H, ArH), 7.70-7.76 (m, 1H, ArH), 7.78-7.88 (m, 2H, ArH) ppm. ${ }^{13} \mathrm{C}$ NMR $\left(\mathrm{CDCl}_{3}, 75 \mathrm{MHz}\right): \delta=36.8,49.9,62.5,66.6,115.4,119.9,120.1,124.6,125.0,126.7,126.8,127.2$, 130.6, 140.6, 142.6, 143.4, 143.7 ppm. ESIMS: $m / z: 291\left(\mathrm{M}^{+1}\right)$.

2-phenyl-2-(phenylamino)propanenitrile (4n): Pale yellow solid; m.p. $138-140{ }^{\circ} \mathrm{C}\left({ }^{\mathrm{Lit}[57]} 133-136{ }^{\circ} \mathrm{C}\right)$. IR (KBr): $\bar{v}=3357,2985,2867,2236,1671,1468,1254, \mathrm{~cm}^{-1} .{ }^{1} \mathrm{H}$ NMR $\left(300 \mathrm{MHz}, \mathrm{CDCl}_{3}\right): \delta=1.72(\mathrm{~s}$, $\left.3 \mathrm{H},-\mathrm{CH}_{3}\right), 4.07(\mathrm{~s}, 1 \mathrm{H},-\mathrm{NH}), 6.31(\mathrm{~d}, 2 \mathrm{H}, J=8.4 \mathrm{~Hz}), 6.56(\mathrm{t}, 1 \mathrm{H}, J=8.2 \mathrm{~Hz}), 6.88(\mathrm{t}, 2 \mathrm{H}, J=8.2 \mathrm{~Hz}$, $\mathrm{ArH}), 7.12-7.21(\mathrm{~m}, 3 \mathrm{H}, \mathrm{ArH}), 7.45(\mathrm{~d}, 2 \mathrm{H}, J=7.4 \mathrm{~Hz}, \mathrm{ArH}) \mathrm{ppm} .{ }^{13} \mathrm{C}$ NMR $\left(75 \mathrm{MHz}, \mathrm{CDCl}_{3}\right): \delta=33.7$, 57.5, 114.0, 120.2, 120.4, 125.1, 128.3, 129.1, 129.5, 139.5, $143.4 \mathrm{ppm}$. ESIMS: $m / z: 222\left(\mathrm{M}^{+1}\right)$

2-((4-methoxyphenyl) amino)-2-phenylpropanenitrile (4o): Pale yellow solid; m.p. 93-95 ${ }^{\circ} \mathrm{C}\left({ }^{\mathrm{Lit}[59]} 88-90\right.$ $\left.{ }^{\circ} \mathrm{C}\right)$. IR (KBr): $\bar{v}=3357,2965,2843,2236,1624,1438,1254 \mathrm{~cm}^{-1} .{ }^{1} \mathrm{H}$ NMR $\left(300 \mathrm{MHz}, \mathrm{CDCl}_{3}\right): \delta=2.40$ (s, $\left.3 \mathrm{H},-\mathrm{CH}_{3}\right), 4.18\left(\mathrm{~s}, 3 \mathrm{H},-\mathrm{OCH}_{3}\right), 4.51(\mathrm{~s}, 1 \mathrm{H},-\mathrm{NH}), 7.04(\mathrm{~d}, 2 \mathrm{H}, J=9.0 \mathrm{~Hz}, \mathrm{ArH}), 7.17(\mathrm{~d}, 2 \mathrm{H}, J=$ 9.2 Hz, ArH), 7.80-7.88 (m, 3H, ArH), $8.10(\mathrm{~d}, 2 \mathrm{H}, J=7.5 \mathrm{~Hz}, \mathrm{ArH}) \mathrm{ppm} .{ }^{13} \mathrm{C}$ NMR $\left(75 \mathrm{MHz}, \mathrm{CDCl}_{3}\right)$ : $\delta=31.4,55.6,58.7,112.3,117.7,125.7,127.8,129.0,137.5,139.9,152.5,158.0$ ppm. ESIMS: $\mathrm{m} / z: 252$ $\left(\mathrm{M}^{+}\right)$

\section{Results and Discussion}

According to our previous studies concerning the use of succinic acid as an efficient acid catalyst for synthesis of 3,4-disubstituted isoxazol-5(4H)-ones. Here, we used succinic acid as catalyst for synthesis of $\alpha$-aminonitriles. In this typical experiment, reaction occurs between aromatic aldehyde or ketone (1), amines (2) and TMS-CN (3) in the presence of succinic acid as an organo-catalyst at room temperature. The reaction was completed within 30 minutes and obtained the corresponding $\alpha$-amino nitriles (4a) derivative were in excellent yields as shown in the general Scheme 1.

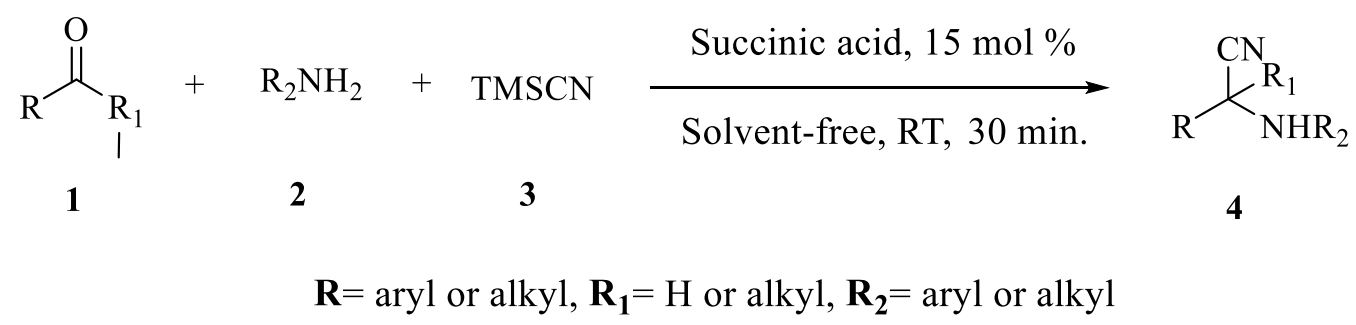

Scheme 1. Synthesis of $\alpha$-amino nitriles catalyzed by succinic acid

To optimize the reaction conditions as well as catalyst, initially the reaction was carried out in the absence of catalyst under solvent free condition but desired product was not observed even extending the reaction time. So the use of catalyst is necessary for improvement of reaction. By considering the importance of catalyst, we chose mild, highly stable, less harmful and commercially cheaply available Succinic acid as catalyst. 
Table 1. Optimization of catalyst

\begin{tabular}{cccc}
\hline No & Succinic acid $(\mathbf{m o l} \%)$ & Time $(\mathbf{m i n})$. & Time $(\mathbf{m i n})$. \\
\hline 1 & No catalyst & 120 & NR* \\
2 & 5 & 30 & 35 \\
3 & 10 & 30 & 55 \\
$\mathbf{4}$ & $\mathbf{1 5}$ & $\mathbf{3 0}$ & $\mathbf{9 0}$ \\
5 & 20 & 30 & 87 \\
\hline
\end{tabular}

$\mathrm{NR}^{*}=$ No Reaction (Product not formed)

Initially, the role of the catalyst was monitored by using different mole ratio from 5-20\% under solvent free condition. The yield of product gradually increases with increasing the amount of catalyst till 15\% mole, but after increasing the amount of catalyst more than $15 \%$ mol, causes gradually decreases the yield of products. The observation shows that $15 \%$ mole equivalent of succinic acid is sufficient for the completion of reaction. All the results are summarized in Table 1.

After optimization of catalyst, we concentrated on screening of solvent system. Initially, the reaction was carried out in water using $15 \%$ mol of catalyst and the corresponding $\alpha$-amino nitrile (4a) was obtained only in $45 \%$ yield. Due to this low yield, we moved to try other solvents systems such as tetrahydrofuran, dichloromethane, acetone, ethanol and yield of corresponding product $\mathbf{4 a}$ obtained with 57, 62, 65 and $70 \%$ respectively. In above all solvents, products yield were somewhat improved but failed to achieve the expected results which is obtained in solvent free condition. The observation shows that a solvent free condition is best reaction condition in terms of the completion of reaction and yield of products. The yield of the product may be increased under solvent free condition due to the greater interaction between the reactant molecules and catalyst. All the results are summarized in Table 2.

Table 2. Optimization of reaction solvent

\begin{tabular}{ccccc}
\hline No & Solvent & Succinic acid (mol \%) & Time (min) & Isolated Yields (\%) \\
\hline 1 & Solvent-free & 15 & 30 & 90 \\
2 & Water & 15 & 30 & 45 \\
3 & Tetrahydrofuran & 15 & 30 & 57 \\
4 & Dichloromethane & 15 & 30 & 62 \\
5 & Acetone & 15 & 30 & 65 \\
6 & Ethanol & 15 & 30 & 70 \\
\hline
\end{tabular}

By encouraged with results obtained in above optimal reaction conditions, the reaction was performed using various aromatic aldehydes and ketones with variety of amines and TMS-CN for the synthesis of $\alpha$-amino nitriles (4a-4o) to demonstrate the applicability of the catalyst (Table 3 ). The feasibility of formation of products and its yields are depends on various electronic as well as steric factors presence on aldehydes or ketones and amines. The aromatic aldehyde having electron withdrawing group obtained in excellent yield while electron donating group gives somewhat decreased yield and in terms of aromatic amines, the results of products yield are vice versa. The reactivity of ketone compared to aldehyde decreases, due to presence of alkyl group, which creates the steric hindrance around carbonyl carbon and due to this steric hindrance; nucleophile does not reach easily at carbonyl carbon for the addition reaction. 
Table 3. Synthesis of $\alpha$-aminonitriles in the presence of succinic acid under solvent-free condition

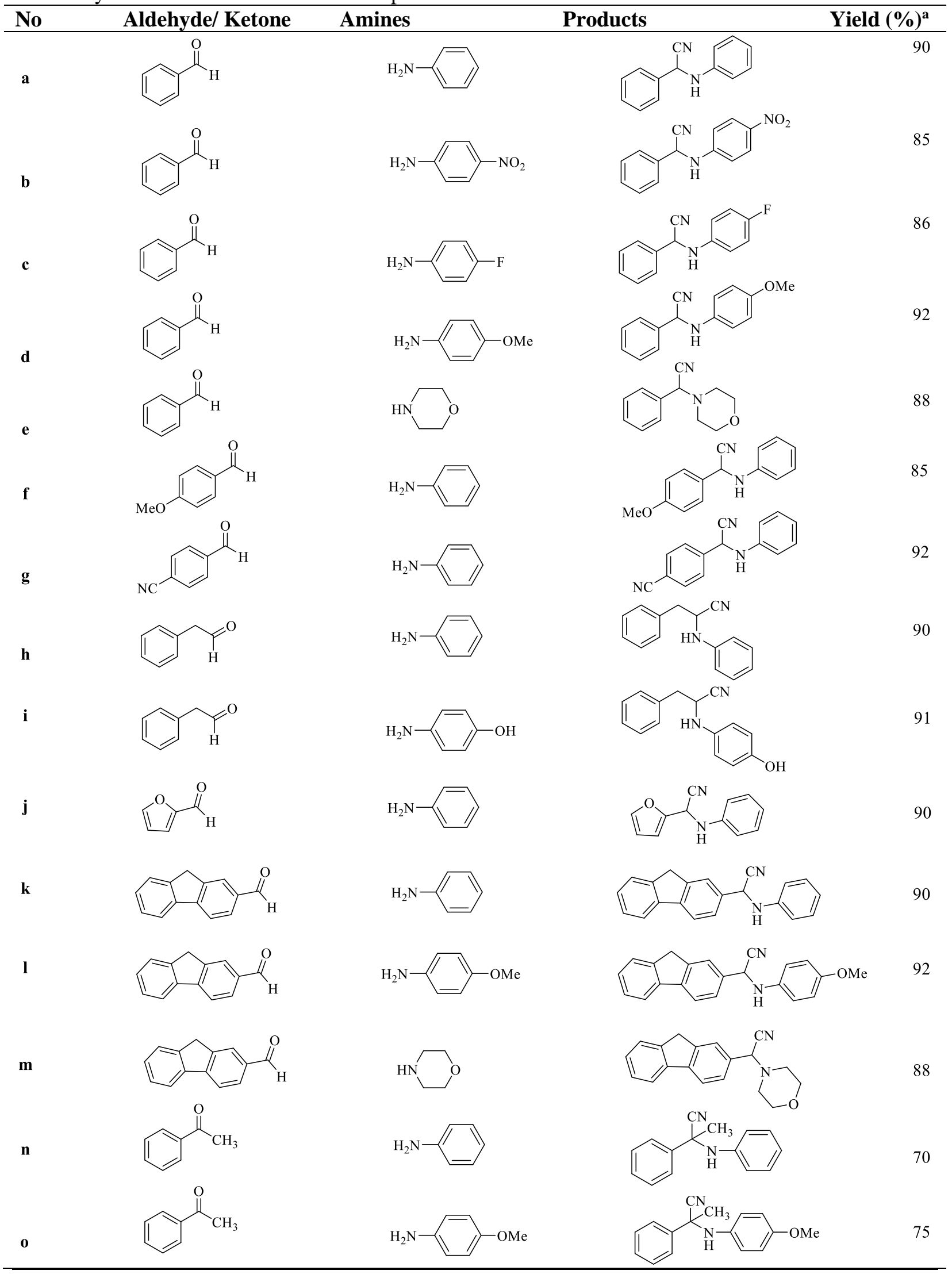


<smiles>O=C(O)CCC(=O)O</smiles>

\section{Succinic acid}

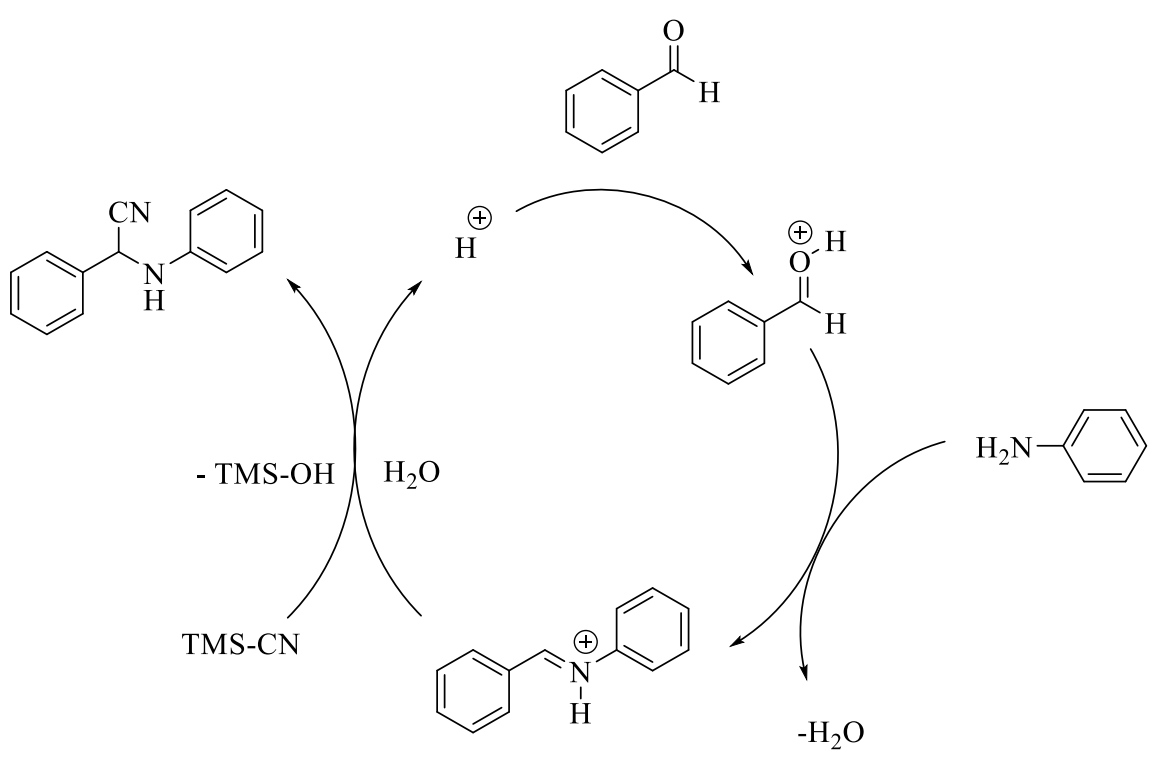

Scheme 2. Probable reaction mechanism

The product formation can be explained as shown in plausible reaction mechanism (Scheme2). The acidic proton of succinic acid has activated the carbonyl carbon of aromatic aldehyde by making coordination with carbonyl oxygen, which leads to formation of imine with aromatic amines. Then the nucleophilic addition reaction occurs between active imines group and trimethylsysil cyanide to formed desired product.

\section{Conclusion}

In summary, we have demonstrated a simple, efficient and novel three-component methodology for the synthesis of Strecker's $\alpha$-amino nitriles by using various aldehyde or ketone, amines and TMS$\mathrm{CN}$. The present method offers significant advantages such as inexpensive catalyst, solvent free, mild reaction conditions, high conversions and excellent yield.

\section{Acknowledgements}

Author is grateful to department of chemistry, B. N. N. College Bhiwandi for providing the laboratory facility.

\section{Supporting Information}

Supporting information accompanies this paper on http://www.acgpubs.org/journal/organic$\underline{\text { communications }}$

\section{ORCID}

Ramesh S. Ghogare: 0000-0003-0810-9636 


\section{References}

[1] Buckley, B. R.; Kimber, M. C.; Slater, N. H. Organocatalysis. Annu. Rep. Prog. Chem., Sect. B: Org. Chem. 2012, 108, 98-109.

[2] Oliveira, V. G.; Cardoso, M. F. C.; Forezi, L. S. M. Organocatalysis: A brief overview on its evolution and applications Catalyst 2018, 8, 605.

[3] MacMillan, D.; The advent and development of organocatalysis. Nature 2008, 455, 304-308.

[4] Dondoni, A.; Massi, A. Asymmetric organocatalysis: From infancy to adolescence. Angew. Chem. Int. Ed. 2008, 47, 4638-4660.

[5] Zhong, C.; Shi, X. When organocatalysis meets transition-metal catalysis. Eur. J.Org. Chem.2010, 2010, 2999-3025.

[6] Govender, T.; Arvidsson, P. I.; Maguire, G. E. M.; Kruger, H. G.; Naicker, T. Enantioselective organocatalyzed transformations of $\beta$-ketoesters. Chem. Rev. 2016, 116, 9375-9437

[7] Delhomme, C.; Weuster-Botz, D.; Kühn, F. E. Succinic acid from renewable resources as a $\mathrm{C}_{4}$ buildingblock chemical-a review of the catalytic possibilities in aqueous media. Green Chem. 2009, 11, 13-26.

[8] Nghiem, N. ; Davison, B. H.; Donnelly, M. I.; Tsai, S. P.; Frye, J. G.; Bozell, J. J. An integrated process for the production of chemicals from biologically derived succinic acid. Am. Chem. Soc. Washington DC. 2001, 784, 160-173.

[9] Mittal, J.; Mathur, R. B.; Bahl, O. P.; Inagaki, M. Post spinning treatment of pan fibers using succinic acid to produce high performance carbon fibers. Carbon 1998, 36, 893-897.

[10] Sacan, L.; Cirpan, A.; Camurlu, P.; Toppare, L. Conducting polymers of succinic acid bis-(2-thiophen-3yl-ethyl)ester and their electrochromic properties. Synth. Met. 2006, 156, 190-195.

[11] Tachibana, Y.; Masuda, T.; Funabashi, M.; Kunioka, M. Chemical synthesis of fully biomass-based poly(butylene succinate) from inedible-biomass-based furfural and evaluation of its biomass carbon ratio. Biomacromolecules 2010, 11, 2760-2765.

[12] Luman, N R.; Kim, T.; Grinstaff, M. W. Dendritic polymers composed of glycerol and succinic acid: Synthetic methodologies and medical applications. Pure Appl.Chem. 2004, 76, 1375-1385

[13] Hazeri, N.; Maghsoodlou, M. T.; Habibi-Khorassani, S. M.; Aboonajmi, J.; Lashkari, M.; Sajadikhah, S. S. A green protocol for one-pot three-component synthesis of a-amino phosphonates catalyzed by succinic acid. Res Chem Intermed. 2014, 40, 1781-1788

[14] Habibi-Khorassani, S. M.; Shahraki, M.; Yaghoubian, H. Insight into the reaction mechanism of $\alpha$-amino phosphonate synthesis using succinic acid as a catalyst: computational kinetic approach. J. Chin. Chem. Soc. 2017, 64,195-204.

[15] Patil, S.; pawar, P. B.; jadhav, S. D.; Deshmukh, M. B. An efficient one-pot multicomponent synthesis of dihydropyridines by using succinic acid as mild organocatalyst. Asian J. Chem. 2013, 25, 9442-9446

[16] Ghogare, R. S.; Patankar-Jain, K.; Momin, S. A. H. A simple and efficient protocol for the synthesis of 3,4-disubstituted isoxazol-5(4H)-ones catalyzed by succinic acid using water as green reaction medium. Lett. Org. Chem. (2020), 17, 1. doi.org/10.2174/1570178617999200721011300

[17] Strecker, A. Ueber die kiinstliche Bildung der Milchsaure und einen neuen, dem glycocoll homologen Korper Ann. Chem. Pharm. 1850, 75, 27-45.

[18] Strecker, A. Ueher einen neuen aus Aldehyd - Ammoniak und Blausiiure entstehenden Kiirper. Ann. Chem. Pharm. 1854, 91, 349-351.

[19] Kouznetsov, V.;V.;Galvis, C. E. P. Strecker reaction and a-amino nitriles: Recent advances in their chemistry, synthesis, and biological properties Tetrahedron 2018, 74, 773- 810

[20] Duthaler, R.O. Recent developments in the stereoelective synthesis of $\alpha$-aminoacids. Tetrahedron 1994, 50, 1539-1650.

[21] Enders, D.; Shilvock, J. P. Some recent applications of $\alpha$-amino nitrile chemistry. Chem. Soc. Rev. 2000, 29, 359-373.

[22] Vedejs, E.; Kongkittingam, C. A total synthesis of (-)-hemiasterlin using N-Bts methodology. J. Org. Chem. 2001, 66, 7355-7364.

[23] Charupant, K.; Daikuhara, N.; Saito, E.; Amnuoypol, S.; $\quad$ Suwanborirux, K.; Owac, T.; Saito, N. Chemistry of renieramycins. Part 8: Synthesis and cytotoxicity evaluation of renieramycin Mjorunnamycin A analogues. Bioorg. Med. Chem. 2009, 17, 4548-4558.

[24] Namba, K.; Shinada, T.; Teramoto, T.; Ohfune, Y. Total synthesis and absolute structure of manzacidin A and C. J. Am. Chem. Soc. 2000, 122, 10708-10709.

[25] Wang, L.; Shen, J.; Tang, Y.; Chen, Y.; Wang, W.; Cai, Z.; Du, Z.; Synthetic Improvements in the Preparation of Clopidogrel. Org. Process Res. Dev. 2007, 11, 487-489. 
[26] Zhang, F.G.; Zhu, X-Y.; Li, S.; Niea, J.; Ma, J.A. Highly enantioselective organocatalytic Strecker reaction of cyclic N-acyl trifluoromethylketimines: synthesis of anti-HIV drug DPC 083. Chem. Commun. (2012), 48, 11552-11554

[27] Dyker, G. Amino acid derivatives by multicomponent reactions. Angew. Chem. Int. Ed.1997,36, 1700-1702.

[28] Enders, D.; Shilvock, J. P. Some recent applications of a-amino nitrile chemistry. Chem. Soc. Rev. 2000, 29, 359-373.

[29] Groger, H. Catalytic enantioselective Strecker reactions and analogous syntheses. Chem. Rev. 2003, 103, 2795-2828.

[30] Gonza'lez-Vera, J. A.; Garcı'a-López, M. T.; Herranz, R. Molecular diversity via amino acid derived ramino nitriles: synthesis of spirocyclic 2,6-dioxopiperazine derivatives. J.Org. Chem. 2005, 70, 36603666.

[31] Ghafuri, H.; Roshani, M. Aqueous formic acid: an efficient, inexpensive and environmentally friendly organocatalyst for three-component Strecker synthesis of $\alpha$-aminonitriles and imines with excellent yields. $R S C A d v$. 2014, 4, 58280-58286.

[32] Vahdat, S. M.; Khaksar, S.; Khavarpour, M. Oxalic acid as a simple and efficient organocatalyst for three-component synthesis of $\alpha$-amino nitriles. Chin Chem Lett. 2011, 22543-22546.

[33] Reddy, Ch. S.; Raghu, M. $p$-Toluenesulphonic acid catalysed rapid and efficient protocol for one-pot synthesis of $\alpha$-amino nitriles. Indian J. Chem. 2008, 47B, 1572-1577.

[34] Das, B.; Kumar, R. A.; Thirupathi, P. One-pot three-component synthesis of $\alpha$-amino nitriles catalyzed by 2,4,6-trichloro-1,3,5-triazine (cyanuric acid). Helvetica Chim. Acta 2007, 90, 1206-1210.

[35] Heydari, A.; Arefi, A.; Khaksar, S.; Shiroodi, R. K. Guanidine hydrochloride: an active and simple catalyst for Strecker type reaction. J. Mol. Catal. A 2007, 271, 142-144.

[36] Nasreen, A. L-Proline catalyzed one pot synthesis of $\alpha$-aminonitriles. Tetrahedron Lett. 2013, 54, 37973800.

[37] Raj, I. V. P.; Suryavanshi, G.; Sudalai, A. Organocatalytic activation of TMSCN by basic ammonium salts for efficient cyanation of aldehydes and imines. Tetrahedron Lett. 2007,48, 7211-7214.

[38] Kaur, G.; Shamim, M.; Bhardwaj, V.; Gupta, V.K.; Banerjee, B. Mandelic acid catalyzed one-pot threecomponent synthesis of $\alpha$-aminonitriles and $\alpha$-aminophosphonates under solvent-free conditions at room temperature. Syn.Commun. 2020, 50, 1545-1560

[39] Chen, W-Y.; Lu, J. Silica sulfuric acid catalyzed one-pot synthesis of $\alpha$-aminonitriles. Synlett. 2005, 15, 2293-2296.

[40] Shekouhy, M. Sulfuric acid-modified PEG-6000 (PEG-OSO3H): an efficient Bronsted acid-surfactant combined catalyst for the one-pot three component synthesis of $\alpha$-aminonitriles in water Catal. Sci. Technol. 2012, 2, 1010-1020.

[41] Shaabani, A.; Maleki, A. Cellulose sulfuric acid as a bio-supported and recyclable solid acid catalyst for the one-pot three-component synthesis of $\alpha$-amino nitriles. Appl. Catal. A Gen. 2007, 331,149 151.

[42] Wang, W.; Wang, Y.; Wu, B. Octahedra-based molecular sieve aluminoborate (PKU-1) as solid acid for heterogeneously catalyzed Strecker reaction. Catal. Commun. 2015, 58, 174-178.

[43] Rahi, T. ; Baghernejad, M.; Niknam, K. Synthesis of $\alpha$-aminonitriles using silica-bonded Npropylpiperazine sulfamic acid as a recyclable catalyst. Chin. Chem. Lett. 2012, 23, 1103-1106.

[44] Dekamin, M. G.; Mokhtari, Z. Highly efficient and convenient Strecker reaction of carbonyl compounds and amines with TMSCN catalyzed by MCM-41 anchored sulfonic acid as a recoverable catalyst. Tetrahedron 2012, 68, 922-930.

[45] Indalkar, K. S.; Khatri, C. K.; Chaturbhuj, G. U. Expeditious and efficient synthesis of Strecker's $\alpha$ aminonitriles catalyzed by sulfated polyborate. Tetrahedron Lett. 2017, 58, 2144-2148.

[46] Kantam, M. L.; Mahendar, K.; Sreedhar, B.; Choudary, B. M. Synthesis of $\alpha$-amino nitriles through Strecker reaction of aldimines and ketoimines by using nanocrystalline magnesium oxide. Tetrahedron 2008, 64, 3351-3360.

[47] Baghbanian, S. M.; Farhang, M.; Baharfar, R. One-pot three-component synthesis of a-amino nitriles catalyzed by nano powder TiO2 P 25. Chin. Chem. Lett. 2011, 22, 555-558.

[48] Reddy, B.M.; Thirupathi, B.; Patil, M.K. Highly efficient promoted zirconia solid acid catalysts for synthesis of $\alpha$-aminonitriles using trimethylsilyl cyanide. J. Mol. Catal. Chem. A 2009, 307, 154-159.

[49] Karimi, B.; Maleki, A.; Elhamifar, D.; Clark, J. H.; Hunt, A. J. Self-assembled organic-inorganic hybrid silica with ionic liquid framework: a novel support for the catalytic enantioselective Strecker reaction of imines using $\mathrm{Yb}(\mathrm{OTf}) 3-$ pybox catalyst. Chem. Commun.

2010, 46, 6947- 6949. 
[50] Eslami, M.; Dekamin, M. G.; Motlagh, L.; Maleki, A. MCM-41 mesoporous silica: a highly efficient and recoverable catalyst for rapid synthesis of $\alpha$-aminonitriles and imines. Green.Chem. Lett. Rev. 2018, 11, 36-46.

[51] Shekouhy, M.; Moaddeli, A.; Khalafi-Nezhad, A. Magnetic $\mathrm{Fe}_{3} \mathrm{O}_{4}-\mathrm{BF}_{3}$ : highly efficient Lewis acid catalyst for the synthesis of a-aminonitriles. Res. Chem. Intermed. 2016, 42, 3805- 3827.

[52] Pamar, M. G.; Govender, P.; Pillay, K.; Abrahamse, H.; Nanjundaswamy, H. M. A fast and benign synthesis of $\alpha$-aminonitriles by reusable immobilized $\mathrm{AlCl}_{3}$ on $\gamma-\mathrm{Al}_{2} \mathrm{O}_{3}$. Indian J. Chem. 2015, 54B, 110116.

[53] Mansoor, S. S.; Aswin, K.; Logaiya, K.; Sudhan. S. P. N. An efficient one-pot three-component synthesis of $\alpha$-amino nitriles via Strecker reaction catalysed by bismuth(III) nitrate. J. Saudi. Chem. Soc.2016, 20, S202-S210.

[54] Majhi, A.; Kim, S. S.; Kadam, S. T. Rhodium(III) iodide hydrate catalyzed three-component coupling reaction: synthesis of $\alpha$-aminonitriles from aldehydes, amines, and trimethylsilyl cyanide. Tetrahedron 2008, 64, 5509-5514.

[55] Ranu, B. C.; Dey, S. S.; Hajra, A. Indium trichloride catlyzed one-step synthesis of $\alpha$-amino nitriles by a three-component condensation of carbonyl compounds, amines and potassiun cyanide. Tetrahedron 2002 , $58,2529-2532$.

[56] Karimi-Jaberi, Z.; Bahrani, A. Boric acid catalysed synthesis of $\alpha$-aminonitriles by a three-component reaction at room temperature. J. Chem. Res. 2012, 36, 326-327.

[57] Hajipoura, A. R.; Ghayeb, Y.; Sheikhan, N. $\mathrm{Zr}\left(\mathrm{HSO}_{4}\right)_{4}$ catalyzed one-pot strecker synthesis of $\alpha$-amino nitriles from aldehydes and ketones under solvent-free conditions. J. Iran. Chem. Soc. 2010, 7, 447-454.

[58] Narasimhulu, M.; Reddy, T, S.; Mahesh, K. C.; Reddy, S. M.; Reddy, A.V.; Venkateshwarlu, Y. Lanthanum(III) nitrate hexahydrate or gadolinium(III) chloride hexahydrate catalyzed one-pot synthesis of $\alpha$-amino nitriles. J. Mol. Catal. Chem. A 2007, 264, 288-292.

[59] Brahmachari, G.; Banerjee, B. A comparison between catalyst-free and $\mathrm{ZrOCl}_{2} \cdot 8 \mathrm{H}_{2} \mathrm{O}$-catalyzed Strecker reactions for the rapid and solvent-free one-pot synthesis of racemic a-aminonitrile derivatives. Asian J. Org. Chem. 2012, 1, 251-258

[60] Karmakar, B.; Banerji, J. $\mathrm{K}_{2} \mathrm{PdCl}_{4}$ catalyzed efficient multicomponent synthesis of $\alpha$-aminonitriles in aqueous media. Tetrahedron Lett. 2010, 51, 2748-2750.

[61] Choi, J.; Yang, H. Y.; Kim, H. J.; Son, S. U. Organometallic hollow spheres bearing bis(N-heterocyclic carbene)-palladium species: catalytic application in three-component Strecker reactions. Angew. Chem. Int. Ed. 2010, 49, 7718-7722.

[62] Jarusiewicz, J.; Choe, Y.; Yoo, K. S.; Park, C. P.; Jung, K. W. Efficient three-component Strecker reaction of aldehydes/ketones via NHC-amidate palladium(II) complex catalysis. J. Org. Chem. 2009, 74, 2873-2876.

[63] Mojtahedi, M. M.; Abaee, M. S.; Abbasi, H. Environmentally friendly room temperature Strecker reaction: one-pot synthesis of $\alpha$-aminonitriles in ionic liquid. J. Iran. Chem. Soc. 2006, 3, 93-97.

[64] Hajipour, A. R.; Dehbanea, I. M. An efficient one-pot synthesis of $\alpha$-amino nitriles using ecofriendly Lewis-acidic ionic liquid choline chloride. $2 \mathrm{ZnCl}_{2 .}$. Iran. J. Catal. 2012, 2, 147-151.

[65] Akbari, J. Synthesis of $\alpha$-amino nitriles through Strecker-type reaction using $\mathrm{SO}_{3} \mathrm{H}$-functionalized ionic liquid as a homogeneous and water tolerant-acidic catalyst. C. R. Chim.2012, 15, 471-473.

[66] Yadav, J. S.; Reddy, B. V. S.; Eshwaraiah, B.; Srinivas, M. P.; Vishnumurthy, P. Three-component coupling reactions in ionic liquids: a facile synthesis of $a$-aminonitriles. New J. Chem. 2003, 27, 462465 .

[67] Sefat, M. N.; Saberi, D.; Niknam, K. Preparation of silica-based ionic liquid an efficient and recyclable catalyst for one-pot synthesis of $\alpha$-aminonitriles. Catal. Lett. 2011, 141, 1713-1720

[68] Prakash, G. K. S.; Thomas, T. E.; Bychinskaya, I.; Prakash, A. G.; Panja, C.; Vaghooa, H.; Olah, G. A. Efficient green synthesis of $\alpha$-aminonitriles, precursors of a-amino acids. Green Chem. 2008, 10, 11051110.

[69] Olah, G. A.; Mathew, T.; Panja, C.; Smith, K.; Prakash, G. K. S. PVP-SO 2 complex as a solid mild acid catalyst for efficient one pot, three component, Strecker synthesis of a)aminonitriles. Catal. Lett. 2007, $114,1-7$

[70] Ghogare, R. S.; Rajeshwari, K.; Narsaiah, A. V. Glycerol mediated Strecker reaction: catalyst-free protocol for the synthesis of $\alpha$-amino nitriles. Lett. Org. Chem. 2014, 11, 688-692.

[71] Martinez, R.; Ramon, D. J.; Yus, M. Catalyst-free multicomponent Strecker reaction in acetonitrile Tetrahedron Lett. 2005, 46, 8471-8474.

[72] S. Ramesh, K. Sivakumar, C. Panja; Arunachalam, P. N.; Lalitha, A. Water-mediated Strecker reaction: an efficient and environmentally friendly approach for the synthesis of $\alpha$-aminonitriles via a three-component condensation. Synth. Commun. 2010, 40, 3544-3551. 
[73] Baeza, A.; Nájera, C.; Sansano, J. M. Solvent-free synthesis of racemic $\alpha$-aminonitriles. Synthesis 2007, 1230-1234.

[74] Harusawa, S.; Hamada, Y.; Shioiri, T. Diethyl phosphorocyanidate(DEPC): a novel reagent for the classicals trecker's@ amino nitriles synthesis. Tetrahedron Lett. 1979, 20, 4663-4666.

[75] Li, Z.; Ma, Y.; Xu, J.; Shi, J.; Cai, H. One-pot three-component synthesis of $\alpha$-aminonitriles using potassium hexacyanoferrate(II) as an eco-friendly cyanide source. Tetrahedron Lett. 2010, 51, 39223926.

[76] Sipos, S.; Jablonkai, I. One-pot synthesis of a-aminonitriles from alkyl and aryl cyanides: a Strecker reaction via aldimine alanes. Tetrahedron Lett. 2009, 50, 1844-1846.

[77] Abdel-Aziz, S.; El-Ahl, A.A. Novel uncatalyzed insertion reaction of in situ formed trichlorosilyl cyanide with imines: a facile silicon mediated synthesis of $\alpha$-aminonitriles. Synth. Commun. 2003, 33, 989-998.

[78] Galletti, P.; Pori, M.; Giacomini, D. Catalyst-free Strecker reaction in water: a simple and efficient protocol using acetone cyanohydrin as cyanide source. Eur. J. Org. Chem. 2011, 2011, 3896-3903.

[79] Wadavrao, S. B.; Ghogare, R. S.; Narsaiah, A. V. A simple and efficient protocol for the synthesis of quinoxalines catalyzed by pyridine. Org. Commun. 2013, 6, 23-30.

[80] Ghogare, R. S.; Narsaiah, A. V.; Glycerin as alternative solvent for the synthesis of thiazoles. Org. Commun.2011, 4, 75-81.

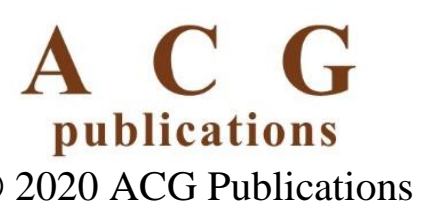

